\title{
Complete genome sequence of Bacteroides salanitronis type strain (BL78 $\left.{ }^{\mathrm{T}}\right)$
}

\author{
Sabine Gronow ${ }^{1}$, Brittany Held ${ }^{2,3}$, Susan Lucas ${ }^{2}$, Alla Lapidus ${ }^{2}$, Tijana Glavina Del Rio ${ }^{2}$, Matt \\ Nolan², Hope Tice ${ }^{2}$, Shweta Deshpande ${ }^{2}$, Jan-Fang Cheng' ${ }^{2}$, Sam Pitluck ${ }^{2}$, Konstantinos \\ Liolios ${ }^{2}$, Ioanna Pagani ${ }^{2}$, Natalia Ivanova ${ }^{2}$, Konstantinos Mavromatis ${ }^{2}$, Amrita Pati ${ }^{2}$, Roxane \\ Tapia $^{2,3}$, Cliff Han ${ }^{2,3}$, Lynne Goodwin ${ }^{2,3}$, Amy Chen ${ }^{4}$, Krishna Palaniappan ${ }^{4}$, Miriam Land ${ }^{2,5}$, \\ Loren Hauser ${ }^{2,5}$, Yun-Juan Chang ${ }^{2,5}$, Cynthia D. Jeffries ${ }^{2,5}$, Evelyne-Marie Brambilla ${ }^{1}$, \\ Manfred Rohde ${ }^{6}$, Markus Göker ${ }^{1}$, John C. Detter ${ }^{2,3}$, Tanja Woyke ${ }^{2}$, James Bristow ${ }^{2}$, Victor \\ Markowitz ${ }^{4}$, Philip Hugenholtz ${ }^{2,8}$, Nikos C. Kyrpides ${ }^{2}$, Hans-Peter Klenk ${ }^{1}$, and Jonathan A. \\ Eisen $^{2,7^{*}}$ \\ ${ }^{1}$ DSMZ - German Collection of Microorganisms and Cell Cultures GmbH, Braunschweig, \\ Germany \\ ${ }^{2}$ DOE Joint Genome Institute, Walnut Creek, California, USA \\ ${ }^{3}$ Los Alamos National Laboratory, Bioscience Division, Los Alamos, New Mexico, USA \\ ${ }^{4}$ Biological Data Management and Technology Center, Lawrence Berkeley National \\ Laboratory, Berkeley, California, USA \\ ${ }^{5}$ Lawrence Livermore National Laboratory, Livermore, California, USA \\ ${ }^{6} \mathrm{HZI}$ - Helmholtz Centre for Infection Research, Braunschweig, Germany \\ ${ }^{7}$ University of California Davis Genome Center, Davis, California, USA \\ ${ }^{8}$ Australian Centre for Ecogenomics, School of Chemistry and Molecular Biosciences, The \\ University of Queensland, Brisbane, Australia \\ *Corresponding author: Jonathan A. Eisen
}

Keywords: strictly anaerobic, non-motile, rod-shaped, Gram-negative, mesophilic, cecum, poultry, chemoorganotrophic, Bacteroidaceae, GEBA

Bacteroides salanitronis Lan et al. 2006 is a species of the genus Bacteroides, which belongs to the family Bacteroidaceae. The species is of interest because it was isolated from the gut of a chicken and the growing awareness that the anaerobic microflora of the cecum is of benefit for the host and may impact poultry farming. The 4,308,663 bp long genome consists of a $4.24 \mathrm{Mbp}$ chromosome and three plasmids ( $6 \mathrm{kbp}, 19 \mathrm{kbp}, 40 \mathrm{kbp}$ ) containing 3,737 proteincoding and 101 RNA genes and is a part of the Genomic Encyclopedia of Bacteria and Archaea project.

\section{Introduction}

Strain BL78 ${ }^{\mathrm{T}}$ (= DSM 18170 = CCUG 54637 = JCM 13657 ) is the type strain of Bacteroides salanitronis which belongs to the large genus Bacteroides $[1,2]$. Currently, there are 88 species placed in the genus Bacteroides. The species epithet is derived from the name of Joseph P. Salanitro, an American microbiologist. B. salanitronis strain $\mathrm{BL78}^{\mathrm{T}}$ was isolated among other Bacteroides strains from the cecum of a healthy chicken. No other strain belonging to the same species has been identified [2]. Many Bacteroides species are common inhabitants of the intestine where they help to degrade complex molecules such as polysaccharides or transform steroids $[3,4]$. They also play a role as beneficent protectors of the gut against pathogenic microorganisms [5]. Here we present a sum- mary classification and a set of features for $B$. salanitronis $\mathrm{BL78}^{\mathrm{T}}$, together with the description of the complete genomic sequencing and annotation.

\section{Classification and features}

A representative genomic 16S rRNA sequence of strain BL78 ${ }^{\mathrm{T}}$ was compared using NCBI BLAST under default settings (e.g., considering only the highscoring segment pairs (HSPs) from the best 250 hits) with the most recent release of the Greengenes database [6] and the relative frequencies, weighted by BLAST scores, of taxa and keywords (reduced to their stem [7]) were determined. The single most frequent genus was Bacteroides (100.0\%) (1 hit in total). Regarding the single hit to sequences from members of the species, the average identity within 
HSPs was $99.7 \%$, whereas the average coverage by HSPs was $96.2 \%$. No hits to sequences with (other) species names were found. The highest-scoring environmental sequence was DQ456041 ('preadolescent turkey cecum clone CFT112F11'), which showed an identity of $96.8 \%$ and an HSP coverage of $63.9 \%$. The five most frequent keywords within the labels of environmental samples which yielded hits were 'fecal' (9.3\%), 'microbiota' (7.5\%), 'human' (7.1\%), 'antibiot, effect, gut, pervas' (7.1\%) and 'anim, beef, cattl, coli, escherichia, feedlot, habitat, synecolog' (2.2\%) (249 hits in total).

Figure 1 shows the phylogenetic neighborhood of $B$. salanitronis in a $16 \mathrm{~S}$ rRNA based tree. The sequences of the six 16S rRNA gene copies in the genome differ from each other by up to 26 nucleotides, and differ by up to 26 nucleotides from the previously published 16S rRNA sequence (AB253731).

The cells of $B$. salanitronis are generally rod-shaped (0.4-0.7 × 0.8-5.6 $\mu \mathrm{m}$ ) with rounded ends (Figure 2). The cells are usually arranged singly or in pairs [2]. $B$. salanitronis is a Gram-negative, non-sporeforming bacterium (Table 1) that is described as non-motile, with only five genes associated with motility having been found in the genome (see below).
The temperature optimum for strain $B L 78^{\mathrm{T}}$ is $37^{\circ} \mathrm{C}$. $B$. salanitronis is a strictly anaerobic chemoorganotroph and is able to ferment glucose, mannose, sucrose, maltose, arabinose, cellobiose, lactose, xylose and raffinose [2]. The organism hydrolyzes esculin but does not liquefy gelatin, and neither reduces nitrate nor produces indole from tryptophan [2]. $B$. salanitronis does not utilize trehalose, glycerol, mannitol, sorbitol or melezitose; rhamnose and salicin are fermented weakly [2]. Growth is possible in the presence of bile [2]. Major fermentation products from broth (1\% peptone, $1 \%$ yeast extract, and $1 \%$ glucose each $(\mathrm{w} / \mathrm{v})$ ) are acetic acid and succinic acid, whereas isovaleric acid is produced in small amounts [2]. B. salanitronis shows activity for alkaline phosphatase, $\alpha$ - and $\beta$-galactosidases, $\alpha$ - and $\beta$ glucosidases, $\alpha$-arabinosidase, leucyl glycine arylamidase, alanine arylamidase and glutamyl glutamic acid arylamidase but no activity for urease, catalase, glutamic acid decarboxylase, arginine dihydrolase, $\beta$-galactosidase 6-phosphate, $\beta$-glucuronidase, $N$ acetyl- $\beta$-glucosaminidase, $\alpha$-fucosidase and arginine, proline, leucine, phenylalanine, pyroglutamic acid, tyrosine, glycine, histidine and serine arylamidase [2].

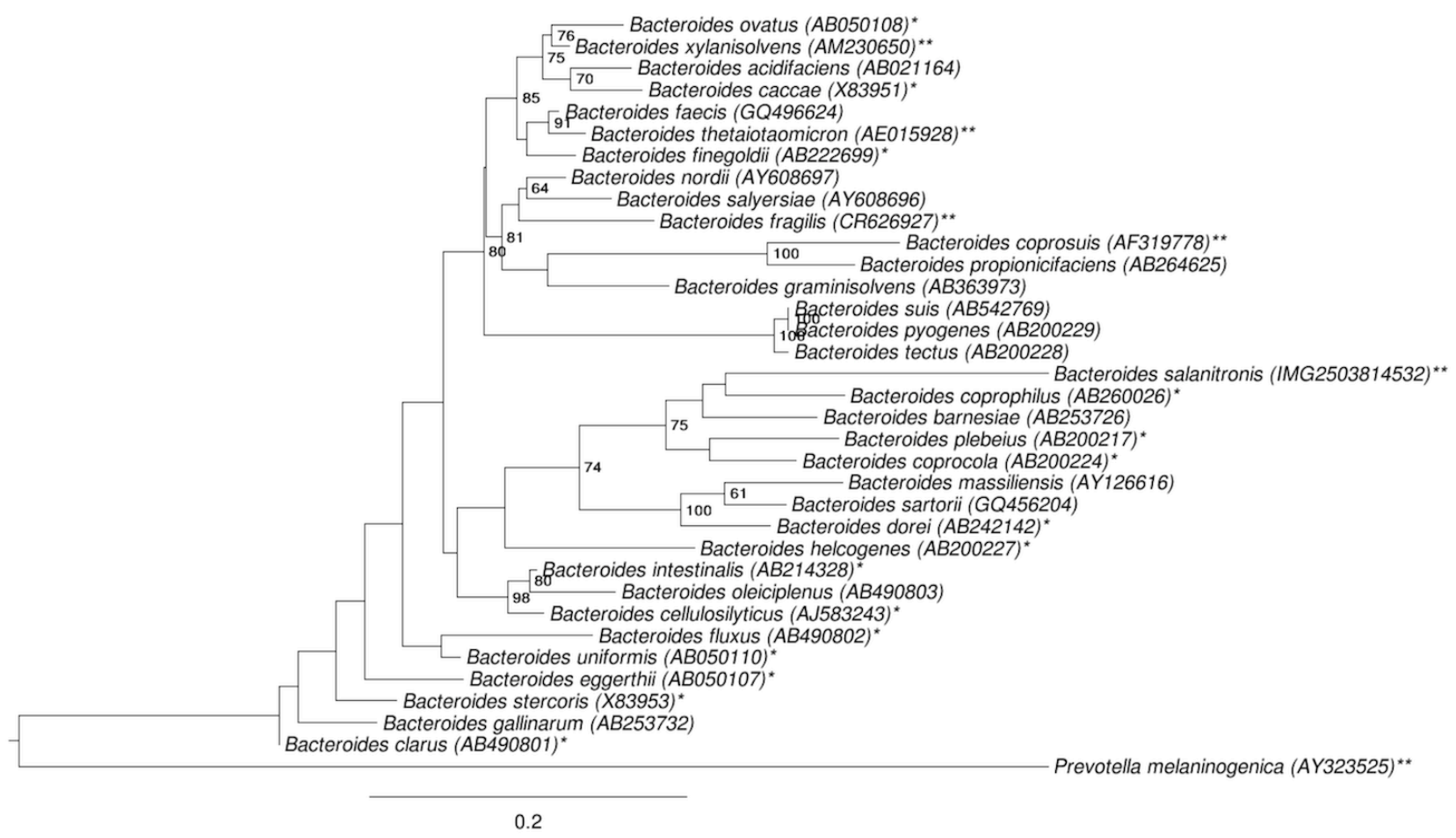

Figure 1. Phylogenetic tree highlighting the position of B. salanitronis relative to a selection of other type strains within the genus. The tree was inferred from 1,412 aligned characters [8,9] of the 16S rRNA gene sequence under the maximum likelihood criterion [10] and rooted in accordance with the current taxonomy. The branches are scaled in terms of the expected number of substitutions per site. Numbers to the right of bifurcations are support values from 1,000 bootstrap replicates [11] if larger than $60 \%$. Lineages with type strain genome sequencing projects registered in GOLD [12] but unpublished are labeled with one asterisk, published genomes with two asterisks [13-15]. 


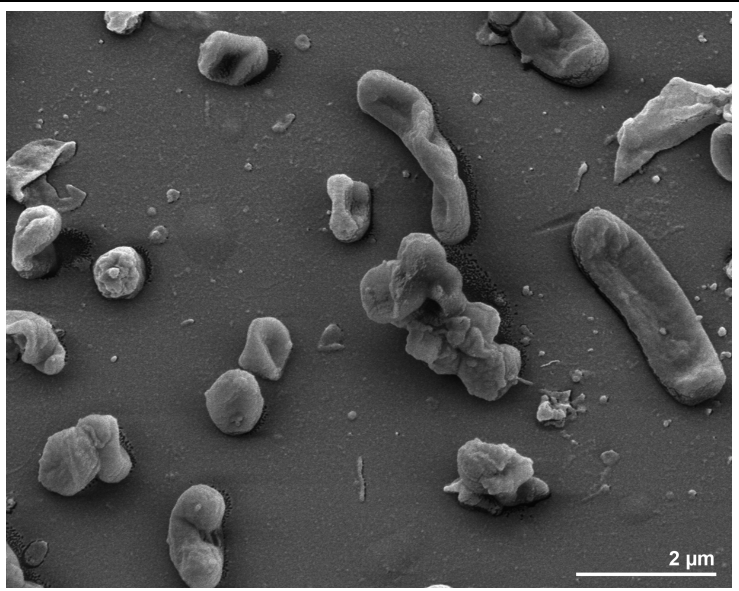

Figure 2. Scanning electron micrograph of $B$. salanitronis $B L 78^{\top}$

Table 1. Classification and general features of B. salanitronis $B L 78^{\top}$ according to the MIGS recommendations [16].

\begin{tabular}{|c|c|c|c|}
\hline MIGS ID & Property & Term & Evidence code \\
\hline & \multirow{8}{*}{ Current classification } & Domain Bacteria & TAS [17] \\
\hline & & Phylum 'Bacteroidetes' & TAS [18] \\
\hline & & Class 'Bacteroidia' & TAS [19] \\
\hline & & Order 'Bacteroidales' & TAS [20] \\
\hline & & Family Bacteroidaceae & TAS $[21,22]$ \\
\hline & & Genus Bacteroides & TAS $[21,23-26]$ \\
\hline & & Species Bacteroides salanitronis & TAS [2] \\
\hline & & Type strain BL78 & TAS [2] \\
\hline & Gram stain & negative & TAS [2] \\
\hline & Cell shape & rod-shaped & TAS [2] \\
\hline & Motility & non-motile & TAS [2] \\
\hline & Sporulation & none & TAS [2] \\
\hline & Temperature range & mesophile & TAS [2] \\
\hline & Optimum temperature & $37^{\circ} \mathrm{C}$ & TAS [2] \\
\hline & Salinity & normal & NAS \\
\hline \multirow[t]{3}{*}{ MIGS-22 } & Oxygen requirement & strictly anaerobic & TAS [2] \\
\hline & Carbon source & carbohydrates & TAS [2] \\
\hline & Energy metabolism & chemoorganotroph & TAS [2] \\
\hline MIGS-6 & Habitat & chicken & TAS [2] \\
\hline MIGS-15 & Biotic relationship & free-living & NAS \\
\hline \multirow[t]{3}{*}{ MIGS-14 } & Pathogenicity & none & NAS \\
\hline & Biosafety level & 1 & TAS [27] \\
\hline & Isolation & chicken cecum & TAS [2] \\
\hline MIGS-4 & Geographic location & Japan & TAS [2] \\
\hline MIGS-5 & Sample collection time & November 2005 & IDA \\
\hline MIGS-4.1 & Latitude & not reported & \\
\hline MIGS-4.2 & Longitude & not reported & \\
\hline MIGS-4.3 & Depth & not reported & \\
\hline MIGS-4.4 & Altitude & not reported & \\
\hline
\end{tabular}

Evidence codes - IDA: Inferred from Direct Assay (first time in publication); TAS: Traceable Author Statement (i.e., a direct report exists in the literature); NAS: Non-traceable Author Statement (i.e., not directly observed for the living, isolated sample, but based on a generally accepted property for the species, or anecdotal evidence). These evidence codes are from of the Gene Ontology project [28]. If the evidence code is IDA, then the property was directly observed by one of the authors or an expert mentioned in the acknowledgements. 


\section{Chemotaxonomy}

B. salanitronis strain $\mathrm{BL}^{2} 8^{\mathrm{T}}$ contains menaquinones MK-11 and MK-12 as principal respiratory quinones (43\% each), small amounts of MK-10 (5\%) and MK-13 (7\%) are found as minor components [2]. The major fatty acids found were anteiso- $\mathrm{C}_{15: 0}$ (32\%), iso- $\mathrm{C}_{15: 0}$ (14\%), 3-hydroxy $\mathrm{C}_{16: 0}$ $(12 \%)$ and 3-hydroxy iso- $\mathrm{C}_{17: 0}(10 \%)$. Fatty acids $\mathrm{C}_{14: 0}(4 \%), \mathrm{C}_{15: 0}(2 \%), \mathrm{C}_{16: 0}(8 \%), \mathrm{C}_{18: 1}(2 \%), \mathrm{C}_{18: 2}$ $(2 \%)$ and iso- $\mathrm{C}_{14: 0}(2 \%)$ were found in minor amounts [2].

\section{Genome sequencing and annotation Genome project history}

This organism was selected for sequencing on the basis of its phylogenetic position [29], and is part of the Genomic Encyclopedia of Bacteria and Archaea project [30]. The genome project is deposited in the Genomes On Line Database [31] and the complete genome sequence is deposited in GenBank. Sequencing, finishing and annotation were performed by the DOE Joint Genome Institute (JGI). A summary of the project information is shown in Table 2.

Table 2. Genome sequencing project information

\begin{tabular}{lll}
\hline MIGS ID & Property & Term \\
\hline MIGS-31 & Finishing quality & Finished \\
MIGS-28 & Libraries used & Three genomic libraries: one 454 pyrosequence standard library, \\
MIGS-31.2 & Sequencing platforms & Illumina GAii, 454 GS FLX Titanium \\
MIGS-30 & Sequencing coverage & $283.0 \times$ Illumina; 37.7 × pyrosequence \\
MIGS-32 & Assemblers & Newbler version 2.3-PreRelease-09-14-2009-bin, Velvet, phrap \\
& Gene calling method & version SPS 4.24 \\
& & Prodigal 1.4, GenePRIMP \\
& INSDC ID & CP002530 (chromosome) \\
& & CP002532 (plasmid 2) \\
& Genbank Date of Release & February 28, 2011 \\
& GOLD ID & Gc001665 \\
& NCBI project ID & 40066 \\
& Database: IMG-GEBA & 2503754023 \\
& Source material identifier & DSM 18170 \\
& Project relevance & Tree of Life, GEBA \\
& &
\end{tabular}

\section{Growth conditions and DNA isolation}

B. salanitronis $\mathrm{BL78}^{\mathrm{T}}$, DSM 18170, was grown anaerobically in DSMZ medium 104 (PeptoneYeast extract-Glucose broth) [32] at $37^{\circ} \mathrm{C}$. DNA was isolated from $0.5-1 \mathrm{~g}$ of cell paste using MasterPure Gram-positive DNA purification kit (Epicentre MGP04100) following the standard protocol as recommended by the manufacturer, adding $20 \mu \mathrm{L}$ lysozyme $(100 \mathrm{mg} / \mu \mathrm{l})$, and $10 \mu \mathrm{L}$ mutanolysin, achromopeptidase, and lysostaphine, each, for 40 min lysis at $37^{\circ} \mathrm{C}$ followed by one hour incubation on ice. DNA is available through the DNA Bank Network [33].

\section{Genome sequencing and assembly}

The genome was sequenced using a combination of Illumina and 454 sequencing platforms. All general aspects of library construction and se- quencing can be found at the JGI website [34]. Pyrosequencing reads were assembled using the Newbler assembler version 2.3-PreRelease-09-142009-bin (Roche). The initial Newbler assembly consisting of 100 contigs in two scaffolds was converted into a phrap assembly [35] by making fake reads from the consensus, to collect the read pairs in the 454 paired-end library. Illumina GAii sequencing data $(920.8 \mathrm{Mb})$ was assembled with Velvet, version 0.7.63 [36] and the consensus sequences were shredded into $1.5 \mathrm{~kb}$ overlapped fake reads and assembled together with the 454 data. The 454 draft assembly was based on 109.0 $\mathrm{Mb}$ of 454 standard data and all of the 454 paired end data. Newbler parameters are -consed -a 50 -l 350 -g -m -ml 20. The Phred/Phrap/Consed software package [35] was used for sequence assem- 
bly and quality assessment in the subsequent finishing process. After the shotgun stage, reads were assembled with parallel phrap (High Performance Software, LLC). Possible mis-assemblies were corrected with gapResolution [34], Dupfinisher [37], or sequencing cloned bridging PCR fragments with subcloning or transposon bombing (Epicentre Biotechnologies, Madison, WI). Gaps between contigs were closed by editing in Consed, by PCR and by Bubble PCR primer walks (J.-F.Chang, unpublished). A total of 193 additional reactions and four shatter libraries were necessary to close gaps and to raise the quality of the finished sequence. Illumina reads were also used to correct potential base errors and increase consensus quality using a software Polisher developed at JGI [38]. The error rate of the completed genome sequence is less than 1 in 100,000. Together, the combination of the Illumina and 454 sequencing platforms provided $320.7 \times$ coverage of the genome. The final assembly contained 393,135 pyrosequence and 25,576,764 Illumina reads.

\section{Genome annotation}

Genes were identified using Prodigal [39] as part of the Oak Ridge National Laboratory genome an- notation pipeline, followed by a round of manual curation using the JGI GenePRIMP pipeline [40]. The predicted CDSs were translated and used to search the National Center for Biotechnology Information (NCBI) nonredundant database, UniProt, TIGR-Fam, Pfam, PRIAM, KEGG, COG, and InterPro databases. Additional gene prediction analysis and functional annotation was performed within the Integrated Microbial Genomes - Expert Review (IMG-ER) platform [41].

\section{Genome properties}

The genome consists of a 4,242,803 bp long chromosome with a $\mathrm{G}+\mathrm{C}$ content of $47 \%$, as well as three plasmids of $6,277 \mathrm{bp}, 18,280 \mathrm{bp}$ and 40,303 bp length (Table 3 and Figure 3). Of the 3,838 genes predicted, 3,737 were protein-coding genes, and 101 RNAs; 96 pseudogenes were also identified. The majority of the protein-coding genes (57.3\%) were assigned with a putative function while the remaining ones were annotated as hypothetical proteins. The distribution of genes into COGs functional categories is presented in Table 4.

Table 3. Genome Statistics

\begin{tabular}{lrr}
\hline Attribute & Value & \% of Total \\
\hline Genome size (bp) & $4,308,663$ & $100.00 \%$ \\
DNA coding region (bp) & $3,759,354$ & $87.25 \%$ \\
DNA G+C content (bp) & $2,003,128$ & $46.49 \%$ \\
Number of replicons & 4 & \\
Extrachromosomal elements & 3 & \\
Total genes & 3,838 & $100.00 \%$ \\
RNA genes & 101 & $2.63 \%$ \\
rRNA operons & 6 & \\
Protein-coding genes & 3,737 & $97.37 \%$ \\
Pseudo genes & 96 & $2.50 \%$ \\
Genes with function prediction & 2,200 & $57.32 \%$ \\
Genes in paralog clusters & 876 & $22.82 \%$ \\
Genes assigned to COGs & 2,013 & $52.45 \%$ \\
Genes assigned Pfam domains & 2,269 & $59.12 \%$ \\
Genes with signal peptides & 918 & $23.92 \%$ \\
Genes with transmembrane helices & 794 & $20.69 \%$ \\
CRISPR repeats & 0 & \\
\hline
\end{tabular}




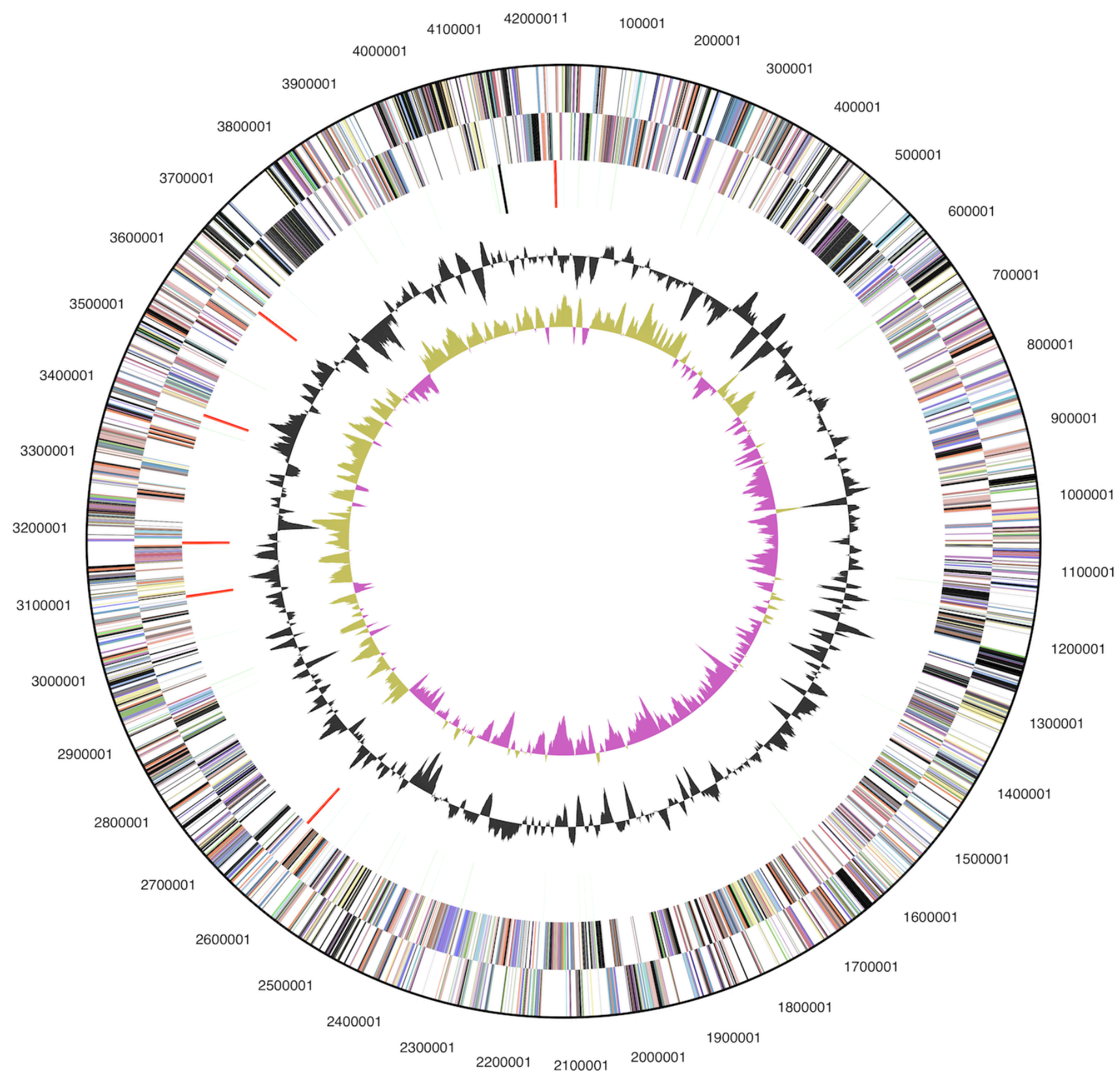

Figure 3. Graphical circular map of the chromosome (plasmid maps not shown). From outside to the center: Genes on forward strand (color by COG categories), Genes on reverse strand (color by COG categories), RNA genes (tRNAs green, rRNAs red, other RNAs black), GC content, GC skew. 
Table 4. Number of genes associated with the general COG functional categories

\begin{tabular}{lrrl}
\hline Code & value & \%age & Description \\
\hline J & 147 & 6.8 & Translation, ribosomal structure and biogenesis \\
A & 0 & 0.0 & RNA processing and modification \\
K & 143 & 6.6 & Transcription \\
L & 194 & 9.0 & Replication, recombination and repair \\
B & 0 & 0.0 & Chromatin structure and dynamics \\
D & 31 & 1.4 & Cell cycle control, cell division, chromosome partitioning \\
Y & 0 & 0.0 & Nuclear structure \\
V & 63 & 2.9 & Defense mechanisms \\
T & 85 & 3.9 & Signal transduction mechanisms \\
M & 193 & 8.9 & Cell wall/membrane/envelope biogenesis \\
N & 5 & 0.2 & Cell motility \\
Z & 0 & 0.0 & Cytoskeleton \\
W & 0 & 0.0 & Extracellular structures \\
U & 61 & 2.8 & Intracellular trafficking, secretion, and vesicular transport \\
O & 61 & 2.8 & Posttranslational modification, protein turnover, chaperones \\
C & 105 & 4.9 & Energy production and conversion \\
G & 174 & 8.0 & Carbohydrate transport and metabolism \\
E & 134 & 6.2 & Amino acid transport and metabolism \\
F & 68 & 3.1 & Nucleotide transport and metabolism \\
H & 98 & 4.5 & Coenzyme transport and metabolism \\
I & 62 & 2.9 & Lipid transport and metabolism \\
P & 104 & 4.8 & Inorganic ion transport and metabolism \\
Q & 29 & 1.3 & Secondary metabolites biosynthesis, transport and catabolism \\
R & 285 & 13.2 & General function prediction only \\
S & 125 & 5.8 & Function unknown \\
- & 1,825 & 47.6 & Not in COGs \\
\hline & & & \\
\hline
\end{tabular}

\section{Acknowledgements}

We would like to gratefully acknowledge the help of Sabine Welnitz (DSMZ) for growing cultures of B. salanitronis. This work was performed under the auspices of the US Department of Energy Office of Science, Biological and Environmental Research Program, and by the University of California, Lawrence Berkeley National Laboratory under contract No. DE-AC02-05CH11231,

\section{References}

1. Garrity G. NamesforLife. BrowserTool takes expertise out of the database and puts it right in the browser. Microbiol Today 2010; 7:1.

2. Lan PTN, Sakamoto M, Sakata S, Benno Y. Bacteroides barnesiae sp. nov., Bacteroides salanitronis sp. nov. and Bacteroides gallinarum sp. nov., isolated from chicken caecum. Int J Syst Evol Micro-
Lawrence Livermore National Laboratory under Contract No. DE-AC52-07NA27344, and Los Alamos National Laboratory under contract No. DE-AC0206NA25396, UT-Battelle and Oak Ridge National Laboratory under contract DE-AC05-000R22725, as well as German Research Foundation (DFG) INST 599/1-2.

biol 2006; 56:2853-2859. PubMed doi:10.1099/ijs.0.64517-0

3. Comstock LE. Importance of glycans to the hostbacteroides mutualism in the mammalian intestine. Cell Host Microbe 2009; 5:522-526. PubMed doi:10.1016/j.chom.2009.05.010 
4. Bäckhed F, Ley RE, Sonnenburg JL, Peterson DA, Gordon JI. Host-bacterial mutualism in the human intestine. Science 2005; 307:1915-1920. PubMed doi:10.1126/science. 1104816

5. Hentges DJ. Role of the intestinal flora in host defense against infection. In Human Intestinal Microflora in Health and Disease 1983; pp. 311331. Edited by D. J. Hentges. New York: Academic Press.

6. DeSantis TZ, Hugenholtz $P$, Larsen $N$, Rojas $M$, Brodie EL, Keller K, Huber T, Dalevi D, Hu P, Andersen GL. Greengenes, a Chimera-Checked $16 \mathrm{~S}$ rRNA Gene Database and Workbench Compatible with ARB. Appl Environ Microbiol 2006; 72:5069-5072. PubMed doi:10.1128/AEM.03006-05

7. Porter MF. An algorithm for suffix stripping. Program: electronic library and information systems 1980; 14:130-137.

8. Lee C, Grasso C, Sharlow MF. Multiple sequence alignment using partial order graphs. BioinformatiCs 2002; 18:452-464. PubMed doi:10.1093/bioinformatics/18.3.452

9. Castresana J. Selection of conserved blocks from multiple alignments for their use in phylogenetic analysis. Mol Biol Evol 2000; 17:540-552.

PubMed

10. Stamatakis A, Hoover P, Rougemont J. A rapid bootstrap algorithm for the RAxML Web servers. Syst Biol 2008; 57:758-771. PubMed doi:10.1080/10635150802429642

11. Pattengale ND, Alipour M, Bininda-Emonds ORP, Moret BME, Stamatakis A. How many bootstrap replicates are necessary? Lect Notes Comput Sci 2009; 5541:184-200. doi:10.1007/978-3-64202008-7_13

12. Liolios K, Chen IM, Mavromatis K, Tavernarakis $\mathrm{N}$, Hugenholtz P, Markowitz VM, Kyrpides NC. The Genomes On Line Database (GOLD) in 2009: status of genomic and metagenomic projects and their associated metadata. Nucleic Acids Res 2010; 38:D346-D354. PubMed doi:10.1093/nar/gkp848

13. Cerdeño-Tárraga AM, Patrick $S$, Crossman LC, Blakely G, Abratt V, Lennard N, Poxton I, Duerden B, Harris B, Quail MA, et al. Extensive DNA inversions in the $B$. fragilis genome control variable gene expression. Science 2005; 307:14631465. PubMed doi:10.1126/science. 1107008

14. Xu J, Bjursell MK, Himrod J, Deng S, Carmichael LK, Chiang HC, Hooper LV, Gordon JI. A genom- ic view of the human Bacteroides thetaiotaomicron symbiosis. Science 2003; 299:2074-2076. PubMed doi:10.1126/science.1080029

15. Land M, Held B, Gronow S, Abt B, Lucas S, Glavina Del Rio T, Nolan M, Tice H, Cheng JF, Pitluck $\mathrm{S}$, et al. Non-contiguous finished genome sequence of Bacteroides coprosuis type strain (PC 139 ${ }^{\mathrm{T}}$. Stand Genomic Sci 2011; 4

16. Field D, Garrity G, Gray T, Morrison N, Selengut J, Sterk P, Tatusova T, Thomson N, Allen MJ, Angiuoli SV, et al. The minimum information about a genome sequence (MIGS) specification. Nat Biotechnol 2008; 26:541-547. PubMed doi:10.1038/nbt1360

17. Woese CR, Kandler O, Wheelis ML. Towards a natural system of organisms: proposal for the domains Archaea, Bacteria, and Eucarya. Proc Natl Acad Sci USA 1990; 87:4576-4579. PubMed doi:10.1073/pnas.87.12.4576

18. Garrity GM, Holt JG. The Road Map to the Manual. In: Garrity GM, Boone DR, Castenholz RW (eds), Bergey's Manual of Systematic Bacteriology, Second Edition, Volume 1, Springer, New York, 2001, p. 119-169.

19. Ludwig W, Euzeby J, Whitman WG. Draft taxonomic outline of the Bacteroidetes, Planctomycetes, Chlamydiae, Spirochaetes, Fibrobacteres, Fusobacteria, Acidobacteria, Verrucomicrobia, Dictyoglomi, and Gemmatimonadetes. http://www.bergeys.org/outlines/Bergeys_Vol_4 Outline.pdf. Taxonomic Outline 2008

20. Garrity GM, Holt JG. 2001. Taxonomic outline of the Archaea and Bacteria, p. 155-166. In G. M. Garrity, D. R. Boone, and R. W. Castenholz (ed.), Bergey's Manual of Systematic Bacteriology, 2nd ed, vol. 1. Springer, New York.

21. Skerman VBD, McGowan V, Sneath PHA. Approved Lists of Bacterial Names. Int / Syst Bacteriol 1980; 30:225-420. doi:10.1099/00207713$\underline{30-1-225}$

22. Pribram E. Klassification der Schizomyceten. Klassifikation der Schizomyceten (Bakterien), Franz Deuticke, Leipzig, 1933, p. 1-143.

23. Castellani A, Chalmers AJ. Genus Bacteroides Castellani and Chalmers, 1918. Manual of Tropical Medicine, Third Edition, Williams, Wood and Co., New York, 1919, p. 959-960.

24. Holdeman LV, Moore WEC. Genus I. Bacteroides Castellani and Chalmers 1919, 959. In: Buchanan RE, Gibbons NE (eds), Bergey's Manual of Determinative Bacteriology, Eighth Edition, The Wil- 
liams and Wilkins Co., Baltimore, 1974, p. 385404.

25. Cato EP, Kelley RW, Moore WEC, Holdeman LV. Bacteroides zoogleoformans, Weinberg, Nativelle, and Prévot 1937) corrig. comb. nov.: emended description. Int I Syst Bacteriol 1982; 32:271-274. doi:10.1099/00207713-32-3-271

26. Shah HN, Collins MD. Proposal to restrict the genus Bacteroides (Castellani and Chalmers) to Bacteroides fragilis and closely related species. Int J Syst Bacteriol 1989; 39:85-87. doi:10.1099/00207713-39-1-85

27. Classification of bacteria and archaea in risk groups. http://www.baua.de TRBA 466.

28. Ashburner M, Ball CA, Blake JA, Botstein D, Butler H, Cherry JM, Davis AP, Dolinski K, Dwight SS, Eppig JT, et al. Gene Ontology: tool for the unification of biology. Nat Genet 2000; 25:25-29. PubMed doi:10.1038/75556

29. Klenk HP, Göker M. En route to a genome-based classification of Archaea and Bacteria? Syst Appl Microbiol 2010; 33:175-182. PubMed doi:10.1016/j.syapm.2010.03.003

30. Wu D, Hugenholtz P, Mavromatis K, Pukall R, Dalin E, Ivanova NN, Kunin V, Goodwin L, Wu $M$, Tindall $B$ J, et al. A phylogeny-driven genomic encyclopaedia of Bacteria and Archaea. Nature 2009; 462:1056-1060. PubMed doi:10.1038/nature08656

31. Markowitz VM, Ivanova NN, Chen IMA, Chu K, Kyrpides NC. IMG ER: a system for microbial genome annotation expert review and curation. Bioinformatics 2009; 25:2271-2278. PubMed doi:10.1093/bioinformatics/btp393

32. List of growth media used at DSMZ: http//www.dsmz.de/microorganisms/media_list.p $\mathrm{hp}$
33. Gemeinholzer B, Dröge G, Zetzsche H, Haszprunar G, Klenk HP, Güntsch A, Berendsohn WG, Wägele JW. The DNA Bank Network: the start from a German initiative. Biopreservation and Biobanking 2011; 9:51-55. doi:10.1089/bio.2010.0029

34. The DOE Joint Genome Institute. http://www.jgi.doe.gov/

35. Phrap and Phred for Windows. MacOS, Linux, and Unix. www.phrap.com

36. Zerbino DR, Birney E. Velvet: algorithms for de novo short read assembly using de Bruijn graphs. Genome Res 2008; 18:821-829. PubMed doi:10.1101/gr.074492.107

37. Han C, Chain P. 2006. Finishing repeat regions automatically with Dupfinisher. In: Proceeding of the 2006 international conference on bioinformatics \& computational biology. Arabina HR, Valafar H (eds), CSREA Press. June 26-29, 2006: 141-146.

38. Lapidus A, LaButti K, Foster B, Lowry S, Trong S, Goltsman E. POLISHER: An effective tool for using ultra short reads in microbial genome assembly and finishing. AGBT, Marco Island, FL, 2008.

39. Hyatt D, Chen GL, LoCascio PF, Land ML, Larimer FW, Hauser LJ. Prodigal: prokaryotic gene recognition and translation initiation site identification. BMC Bioinformatics 2010; 11:119. PubMed doi:10.1186/1471-2105-11-119

40. Pati A, Ivanova NN, Mikhailova N, Ovchinnikova G, Hooper SD, Lykidis A, Kyrpides NC. GenePRIMP: a gene prediction improvement pipeline for prokaryotic genomes. Nat Methods 2010; 7:455-457. PubMed doi:10.1038/nmeth.1457

41. Markowitz VM, Ivanova NN, Chen IMA, Chu K, Kyrpides NC. IMG ER: a system for microbial genome annotation expert review and curation. Bioinformatics 2009; 25:2271-2278. PubMed doi:10.1093/bioinformatics/btp393 